\title{
Vulvar Fibroadenoma: Case Report and Review of Literature
}

\author{
Chalapathi Rao $\mathrm{AV}^{1}$, Srikanth $\mathrm{U}^{2}$, and Haqq $\mathrm{W}^{3}$ \\ ${ }^{1}$ Senior Lecturer/Consultant Pathologist, HOD Paraclinical Sciences, The University of the West Indies, St. Augustine, Trinidad and \\ Tobago \\ ${ }^{2}$ Lecturer/Consultant Pathologist, The University of the West Indies, St.Augustine, Trinidad and Tobago \\ ${ }^{3}$ Consultant in Obstetrics and Gynaecology, Mount Hope Women's Hospital, Champs Fleurs, Trinidad and Tobago
}

*Corresponding author: Srikanth U, Lecturer/Consultant Pathologist, The University of the West Indies, St. Augustine, Trinidad and Tobago, Tel: 1-868-645-2645 Ext: 2330, E-mail: Srikanth.Umakanthan@sta.uwi.edu

Citation: Chalapathi Rao AV, Srikanth U, Haqq W (2017) Vulvar Fibroadenoma: Case Report and Review of Literature. SAJ Case Reports 4: 204

Article history: Received: 31 January 2017, Accepted: 15 March 2017, Published: 17 March 2017

\begin{abstract}
Vulvar fibroadenoma is an uncommon benign tumor with few reported cases in literature. The histogenesis of this tumor has remained controversial. We report a case of a 39 year old woman with a well-defined subcutaneous mass in the vulva with a histological diagnosis of Vulvar fibroadenoma and positive for hormone receptors.
\end{abstract}

Keywords: Vulva; Fibroadenoma; Mammary Gland

\section{Introduction}

Vulvar fibroadenoma is an extremely rare benign tumor with low incidence. This tumor has been reported in medical literature with 50 cases of ectopic benign breast lesion. The milk line which extends from the axilla to the medial aspect of the groin undergoes spontaneous regression during embryogenesis except in the region of the chest giving rise to breasts in adults. Accessory breast structures arise from these remnants when they fail to regress. These are mainly seen in the pectoral region and rarely in vulvar site [1]. Vulvar fibroadenomas occur between 20- 60 years with a size ranging from $0.7 \mathrm{~cm}$ to $6.0 \mathrm{~cm}$. The differential diagnosis ranges from benign lesions like epidermal cyst, Bartholin's cyst, phyllodes to malignant lesions like extramammary Paget's disease and mucinous adenocarcinoma. Since these ectopic mammary tissue is hormonal responsive [2]. Immunohistochemically, these tumor show positivity for ER, PR and GCDF-15. S- 100, CK and EMA are also positive. The vulvar fibroadenoma can also be diagnosed by fine needle aspiration cytology (FNAC). We discuss a case of vulvar fibroadenoma in a 39 year old woman.

\section{Case Report}

A 39 year old G5 P4 +1 woman presented with a painless firm vulvar swelling of 6 months. The swelling was initially small and grew progressively in size. Physical examination revealed a $3 \mathrm{~cm}$ x $2 \mathrm{~cm}$ mass located in the subcutaneous area of the right labium majus. The swelling was firm, mobile, and not adherent to skin or other underlying structures. Gynaecological, inguinal and abdominal examination was reported normal. Chest x-ray and Computed tomography (CT) scan of the abdominopelvic region revealed no masses.

A provisional clinical diagnosis of Bartholin cyst was made and excision was planned. The lesion was excised under local anaesthetic and sedation. During surgery, the lesion was found to be firm, mobile and well defined. The patient was discharged the following day. The patient failed to follow up.

The excised specimen on macroscopic examination showed a firm mass measuring 2.5 x 1.5 x $0.7 \mathrm{cms}$. Cut surface was greywhite. There was no cyst. Areas of haemorrhage and necrosis were absent. Microscopy showed epithelial and stromal proliferation. Glandular elements were benign and ranged from small rounded gland like structures to branched ducts and displayed myoepithelial cells. There was no evidence of atypia. Fibro collagenous stroma showed mild increase in cellularity in the periglandular areas (Figure $1 \mathrm{~A}$ and B). Immunohistochemical studies were done and the glandular cells showed strong positivity for ER and PR (Figure 2A and B). These histopathological findings were of vulvar fibroadenoma. 


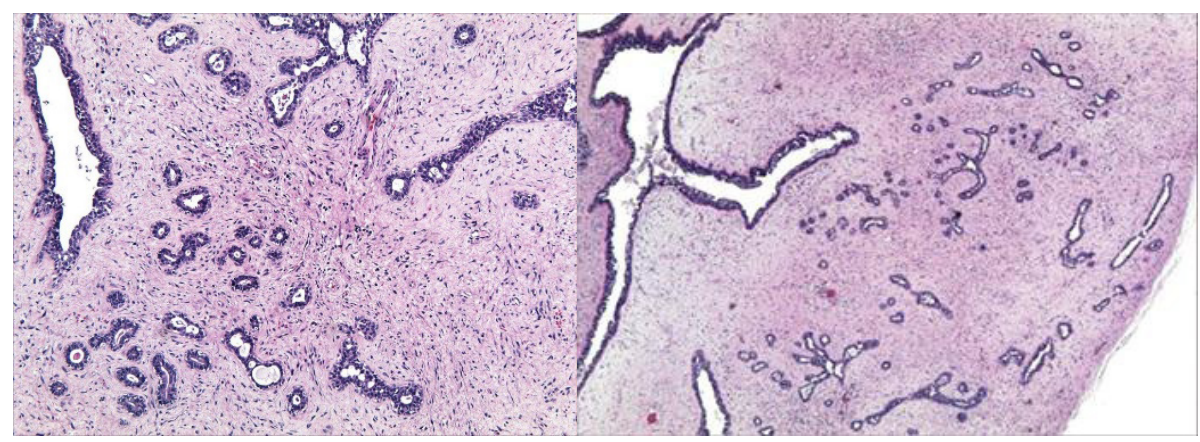

Figure 1 A and B: (H\&E x10) Microscopic picture showing glandular and stromal components of fibroadenoma

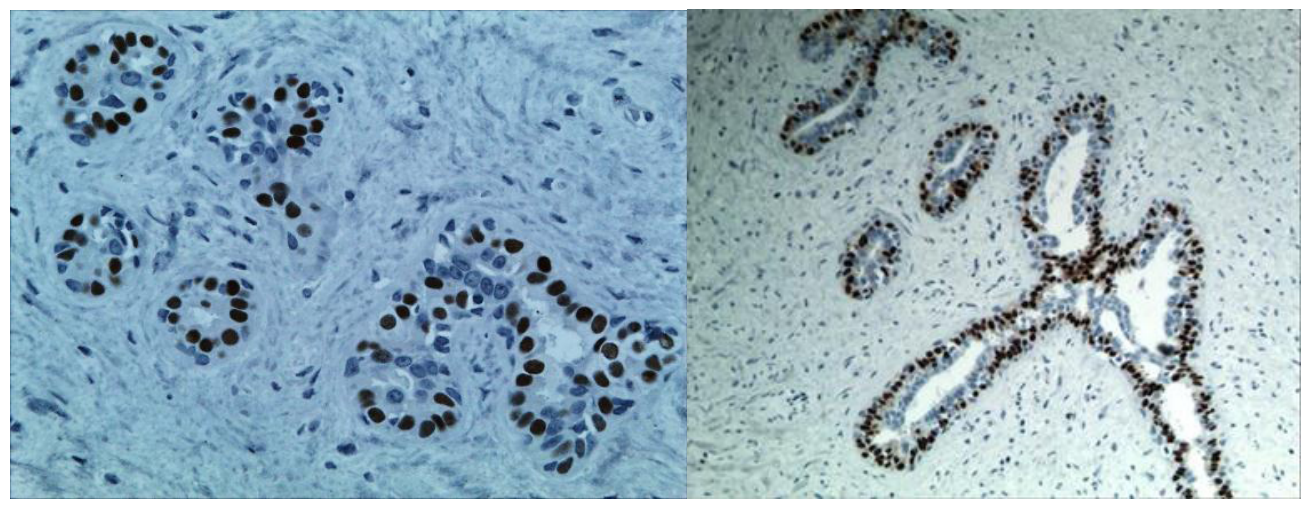

Figure 2: (A) Ductal cells with Estrogen receptor positivity ( $\mathrm{x} 40)$ and (B) progesterone receptor positivity $(20 \mathrm{x})$

\section{Discussion}

The fully formed mammary gland in the vulva was first described by Hartung in 1872 . The histogenesis of vulvar fibroadenoma is controversial. Vulva is a rare site for ectopic breast tissue since it is located at the inferior end of the primitive mammary ridge. Several forms of accessory breast has been classified by Kajava which depends on the presence of breast structures (nipple, areola, glandular tissue) and ranges from complete breast with nipple, areola and glandular tissue to polythelia pilosa (presence of only patch of hair). Japanese women have the highest incidence of accessory mammary tissue [3].

Van der Putte in his first report titled "mammary tissue of anogenital area" discussed that the extension of mammary ridge to the vulva has not been proved definitely and the theory of milk ridge cannot explain perineal ectopic breast tumors. The ectopic breast tissue or mammary like anogenital glands tissue express hormone receptors which are detected by immunohistochemistry [4].

The vulvar fibroadenoma is seen in the age group ranging from 20-60 years with an average tumor size of $3.0 \mathrm{cms}$. They present as subcutaneous solitary nodules on labia majora and are seldom bilaterally seen. The differential diagnoses are epidermal cyst, follicular cyst, Bartholin's gland duct cyst, lipoma, pseudoangimatous stromal hyperplasia, fibrocystic disease and sclerosing adenosis. Extra mammary Paget's disease, ductal/lobular/mucinous adenocarcinoma are malignant differentials [2,3]. Histopathological features are similar to breast fibroadenoma showing a well-defined capsule, epithelial and stromal proliferation with compressed glands and fibromyxoid stroma surrounding it. The glands are lined by luminal columnar cells and abluminal myoepithelial cells [5]. In our case, histopathological features showed features of fibroadenoma with immunohistochemistry positive for ER and PR receptors. The vulvar fibroadenoma has a good prognosis and rarely recurs following excision. In our case the patient failed to follow up [6].

Vulvar fibroadenoma is a rare benign tumor with a controversial histogenesis, arising either from a vulvar accessory breast or from mammary like anogenital glands described by van der Putte.

\section{Declaration of interest}

The authors report no conflicts of interest. The authors alone are responsible for the content and writing of the paper.

\section{Ethics and Consent}

Our study is according to the ethics principles in the Declaration of Helsinki.

\section{References}

1. Cantú de Leon D, Perez Montiel D, Vázquez H, Hernández C, Cetina L, et al. (2009) Vulvar fibroadenoma: a common neoplasm in an uncommon site World J Surg Oncol 7: 70.

2. Pathak S, Preston J (2007) A rare case of multiple accessory breast tissue in the axillae, lower abdomen and vulval areas. J Obstet Gynaecol 27: 531-3. 
3. Baker GM, Selim MA, Hoang MP (2013) Vulvar adnexal lesions: a 32-year, single-institution review from Massachusetts General Hospital. Arch Pathol Lab Med 137: 1237-46.

4. CC Anunobi, FJN Obiajulu, AAF Banjo, and AOE Okonkwo (2013). Vulval fibroadenoma associated with lactating adenoma in a 26 year old Nigerian female. Case Rep Pathol 2013: 195703.

5. Amen Dhaoui, Haifa Nfoussi, Nidhameddine Kchir, and Slim Haouet (2012). Vulvar lactating adenoma associated to a fibroadenoma: common neoplasms in an uncommon site. Pan Afr Med J 13: 47.

6. Lucas EW Jr, Branton P, Mecklenburg FE, Moawad GN (2009). Ectopic breast fibroadenoma of the vulva. Obstet Gynecol 114: 460-2. 